\title{
Repensando los límites de las organizaciones por medio de la teoría de sistemas organizacionales de Niklas Luhmann
}

\author{
Rethinking Organizational Boundaries via Luhmann's Theory of Organized Systems
}

M. Pilar Opazo

Escuela de Negocios, Universidad de Columbia, Estados Unidos

Darío Rodríguez

Escuela de Ingeniería Industrial, Universidad Diego Portales, Chile

RESUMEN

Este artículo examina el modo en que la teoría de sistemas organizados de Luhmann puede servir para ampliar nuestra comprensión de los límites organizacionales. Revisamos, de manera sistemática, cuatro componentes de la obra de Luhmann: i) su distinción básica entre organización y entorno, ii) la apertura y clausura del funcionamiento de la organización, iii) el papel de las comunicaciones y decisiones en la identificación de los límites de las organizaciones y, finalmente, iv) el énfasis que pone Luhmann sobre la coordinación en la construcción de los límites organizacionales. Discutimos los puntos más destacados tanto de divergencia como de correspondencia entre el trabajo de Luhmann y otros enfoques teóricos competidores para entender las organizaciones. Para concluir, se discuten contribuciones potenciales de la teoría luhmanianna para análisis, tanto empíricos como teóricos, de las organizaciones contemporáneas.

PALABRAS ClAVE: Organizaciones; Límites organizacionales; Teoría sistémica; Redes sociales; Niklas Luhmann

ABSTRACT

This article examines how Luhmann's theory of organized systems can be used to expand our understanding of organizational boundaries. In a systematic way, we examine four central components of Luhmann's work: i) its basic distinction between organization and environment, ii) the openness and closeness of the organizations' functioning, iii) the role of communications and decisions in identifying the limits of organizations and, lastly, iv) Luhmann's emphasis on coordination in the construction of organizational boundaries. We discuss the salient points of both divergence and correspondence between Luhmann's work and contending theoretical approaches to organizations. Potential contributions of Luhmann's theory to analyses of contemporary organizations, both empirical and theoretical, are discussed.

KEYWORDS: Organizations; Organizational boundaries; Systems theory; Social networks; Niklas Luhmann

\section{INTRODUCCIÓN}

La construcción de una identidad organizacional depende críticamente de su capacidad para mantener sus límites, vale decir, de marcar su distinción respecto a su entorno externo (White 2008). Del mismo modo, la "demarcación de límites" [boundary-making] (Gieryn 1983) realizada por las or-

\footnotetext{
REVISTA MAD - REVISTA DEL MAGÍSTER EN ANÁLISIS SISTÉMICO APLICADO A LA SOCIEDAD ISSN 0718-0527

Facultad de Ciencias Sociales, Departamento de Antropología.

Universidad de Chile

www.revistamad.uchile.cl
}

DOI: 10.5354/0718-0527.2017.46140 
ganizaciones es clave para entender su funcionamiento. En este artículo reexaminamos la forma en que se ha pensado teóricamente el tema de los límites organizacionales en la literatura académica, utilizando como apoyo el instrumental analítico propuesto por el sociólogo Niklas Luhmann. De manera sistemática, discutimos cuatro elementos clave de la propuesta teórica de Luhmann y exploramos su distinción respecto a otros enfoques habitualmente usados en estudios organizacionales, tales como el institucionalismo o el de redes sociales.

El trabajo de Luhmann constituye una de las teorías sociológicas más comprehensivas de la sociedad contemporánea. Hasta el momento, hay ejemplos notables de trabajos académicos construidos con el apoyo de Luhmann para referirse a temas relacionados con los límites organizacionales desde una perspectiva empírica y teórica (Lane \& Bachmann 2002; Schreyögg \& Seidl 2005; Seidl \& Becker 2005, 2006; Hernes 2007; Sydow 2010; Mohe \& Seidl 2011). No obstante, a pesar de estas significativas contribuciones, el trabajo de Luhmann sigue estando en gran medida sub-considerado por los especialistas organizacionales.

La falta de ejemplos empíricos incluidos en la teoría de Luhmann hace difícil para los especialistas comprender sus conceptos y, más aun, encontrar formas de aplicarlos en sus análisis de organizaciones del mundo real. Por lo tanto, sintetizamos y discutimos los elementos centrales del trabajo de Luhmann. Lo hacemos aclarando sus conexiones y desconexiones con las teorías conocidas de las organizaciones, mediante la presentación de una definición clara de la terminología luhmanniana y usando ejemplos de estudios empíricos de las organizaciones siempre que sea posible. El objetivo de nuestro análisis es doble: en primer lugar, dilucidar aún más las formas en que la obra de Luhmann podría contribuir al avance de la investigación teórica y empírica sobre las organizaciones contemporáneas y, en segundo lugar, poner de relieve las oportunidades para reconsiderar las ventajas y limitaciones de los enfoques analíticos existentes -tanto del propio Luhmann como de otros especialistascuando se trata de examinar el papel que desempeñan los límites organizacionales para comprender la supervivencia y el desarrollo de las organizaciones.

\section{ORGANIZACIÓN Y ENTORNO}

Según Luhmann, las organizaciones surgen y se reproducen al establecer sus diferencias respecto a su entorno. Lo explica haciendo uso del trabajo de Spencer Brown "Las leyes de la forma" (1979) que señala que una distinción es una forma de dos lados, en la cual uno de los lados es indicado y el otro permanece sin ser marcado. Dice Luhmann que no es posible observar ambos lados simultáneamente. Así, la única forma en que una organización puede observarse a sí misma es reintroduciendo una nueva distinción al interior de la distinción original (re-entry), en otras palabras, transformándose en un "observador de segundo orden" (von Foerster 
Repensando los límites de las organizaciones por medio de la teoría de sistemas organizacionales de

Niklas Luhmann

1981). Luhmann explica que, dado que las organizaciones no pueden liberarse de esta primera distinción, esta se mantiene como un punto ciego, no marcado (unmarked space). También hace notar que esto no es un problema para las organizaciones; sino, más bien, es el único modo en que su auto-observación es posible (Luhmann 2010).

Este postulado básico del trabajo de Luhmann es importante, porque ilustra una paradoja irresoluble ubicada en el centro de la teorización organizacional: ni la organización ni su entorno pueden ser entendidos sin el respectivo otro, porque representan dos lados de la misma distinción (Luhmann 1994, 1995, 2010). En palabras de Luhmann, "El sistema no es ontológica ni analíticamente más importante que el entorno; ambos son lo que son solo en referencia mutua" (Luhmann 1995: 173). Tempranos estudios empíricos de organizaciones, tales como los de Selznick (1953) o Burns y Stalker (1961), revelaron esta distinción ontológica al demostrar que no es posible entender las actividades internas de las organizaciones sin dar cuenta de lo que está sucediendo en sus respectivos entornos.

Sin embargo, la conceptualización de Luhmann también enfatiza otro aspecto de las organizaciones que es aún más importante en los estudios de las organizaciones contemporáneas que operan en entornos rápidamente cambiantes (Brown \& Eisendhardt 1998). El hecho de que las organizaciones solo puedan describirse a sí mismas como observadores de segundo orden implica que la unidad de las organizaciones solo puede ser concebida como oscilación, vale decir, como procesos continuos de revelar y ocultar. La identidad de una organización, entonces, es cristalizada y sostenida mediante procesos de trazado de límites.

Esta premisa de la teoría de Luhmann tiene significativas repercusiones para el estudio de las organizaciones, porque implica que el "trabajo de límites" llevado a cabo por las organizaciones no es solamente un aspecto de la operación organizacional, sino el proceso esencial por el cual las organizaciones construyen su identidad y son capaces de perdurar. Esta premisa también llama nuestra atención sobre la importancia de la agencia de las organizaciones al construir su propia representación de su entorno, algo que, como veremos en seguida, es frecuentemente dado por obvio en el análisis contemporáneo de las organizaciones.

\section{APERTURA Y CLAUSURA: LAS ORGANIZACIONES COMO SISTEMAS AUTOPOIÉTICOS}

Richard Scott (2004) describe el surgimiento de la "teoría de sistemas abiertos", durante la década de los 1960s, como uno de los más importantes cambios en la sociología organizacional. De hecho, desde este periodo en adelante, los estudios de organizaciones han incluido al entorno y los procesos de construcción de límites llevados a cabo por organizaciones como factores cruciales en sus análisis, y lo han hecho de diferentes 
maneras. Los enfoques económicos han centrado sus explicaciones en la capacidad de una organización para gestionar transacciones en formas efectivas de costos, enfatizando así la toma de decisiones interna de una organización en su relación con su entorno (Williamson 1981; Williamson \& Ouchi 1981). Los enfoques institucionales se han focalizado más bien en el lado opuesto de la distinción, al observar las formas en que las organizaciones son conformadas por las reglas categóricas o "mitos" que operan en sus entornos (Meyer \& Rowan 1977; DiMaggio \& Powell 1993). Por otra parte, mientras los teóricos de poder-dependencia afirmaban que lo más importante era el control logrado por las organizaciones sobre recursos limitados (Pfeffer \& Salancik 1978), los enfoques de ecología de poblaciones proponían la capacidad de una organización para competir por recursos, como el principal determinante de la supervivencia y duración de una organización (Hannan \& Freeman 1977, 1993).

Sugerimos que, al incorporar el concepto de "autopoiesis", la teoría de Luhmann ofrece una oportunidad para moverse más allá del paradigma de sistemas abiertos en la teorización de la mantención de límites de las organizaciones. Los sistemas autopoiéticos son definidos como sistemas que, en su operación, producen los elementos que los constituyen (Maturana \& Varela 1998). Poiesis significa la producción de un producto; autopoiesis significa la autoproduccion de un sistema como su propio producto (Luhmann 2010). Lo importante en este concepto es que enfatiza tanto la clausura de una organización -que Luhmann llamó clausura operativa- como la apertura de la organización a su entorno -que él definió como acoplamiento estructural- en cuanto dimensiones igualmente importantes para entender el trabajo de las organizaciones (Febbrajo \& Harste 2013). Este concepto ha sido recientemente revitalizado por los especialistas organizacionales Padgett y Powell (2012) quienes usaron el término de sonido similar, "autocatálisis," para explicar el funcionamiento de las organizaciones.

\subsection{Clausura operativa}

De acuerdo con Luhmann, en sus procesos de construcción de límites, las organizaciones hacen un mapa de sus entornos de una forma particular: las organizaciones solo ven lo que han notado y dejan de ver lo que han dejado sin marcar. Un importante corolario de esto es que el entorno percibido por una organización $-\mathrm{y}$, por consiguiente, en el cual ella funciona en la práctica- es el resultado de su propia operación, una pintura que la misma organización crea y recrea de manera constante.

A la luz del concepto de clausura operativa de Luhmann, adquieren un nuevo e interesante sentido nociones propuestas por otros especialistas organizacionales tales como la "organización que aprende" (Senge 1990), "desviación organizacional" (Vaughan 1999), "performatividad" en organizaciones (MacKenzie et.al 2007; Elyachar 2005) o los procesos de "generación de sentido" (sense-making) desarrollados por individuos al 
interior de organizaciones (Weick 1979, 1995). Si consideramos que las organizaciones elaboran un cuadro de su entorno para darle un sentido, de acuerdo a su propia delineación de límites, entonces emerge una explicación totalmente diferente sobre el porqué las organizaciones a menudo mal interpretan sus entornos o fracasan en reaccionar rápidamente a ciertas situaciones. Al respecto, estimamos que, sin el concepto de clausura operacional, el paradigma de sistemas abiertos no alcanza a reconocer la importancia de los límites organizacionales, así como tampoco la agencia de las organizaciones en el trazado de estos límites, vale decir, la capacidad de la organización de producir y reproducir su propia identidad, modelar su entorno y ser modelada por el mismo. También el paradigma de sistemas abiertos fracasa al dar cuenta de la construcción que hace el sistema de su propia pintura del entorno para estar en condiciones de interpretarlo. Para decirlo en una sola frase, el marco analítico de Luhmann enfatiza que hay tantos entornos -y mapas de entornos- como organizaciones.

\subsection{ACOPLAMIENTO ESTRUCTURAL}

A diferencia del modelo de sistemas abiertos, el enfoque de Luhmann afirma que es precisamente debido a que las organizaciones son clausuradas operativamente, que pueden ser abiertas a sus entornos $\mathrm{y}$, por lo tanto, pueden estar acopladas estructuralmente a dichos entornos. La clausura operativa es un prerrequisito de la apertura. Desde la teoría de Luhmann, por lo tanto, es inviable la metáfora de la "organización sin límites" propuesta en estudios de organizaciones (Ashkenas 2002). Si una organización fracasa al preservar sus límites, esto es, su diferencia respecto a su entorno, simplemente cesa de existir como tal. En palabras de Luhmann, "la mantención de límites es la mantención del sistema" (Luhmann 1995: 17, cursiva en el original).

En resumen, la noción de los sistemas autopoiéticos de Luhmann nos permite referirnos explícitamente a la capacidad de una organización para preservar sus límites (clausura operativa) y explotar su relación con su entorno (acoplamiento estructural). En tanto la importancia de esta dualidad puede pasar desapercibida por el "modelo de sistemas abiertos", lo opuesto ocurre si consideramos el modelo de Luhmann de las organizaciones como sistemas autopoiéticos. En el enfoque analítico de Luhmann, tanto la clausura como la apertura de las organizaciones crean la condición de posibilidad de la existencia de éstas. 
FIGURA 1: MODELOS SISTÉMICOS DE LAS ORGANIZACIONES
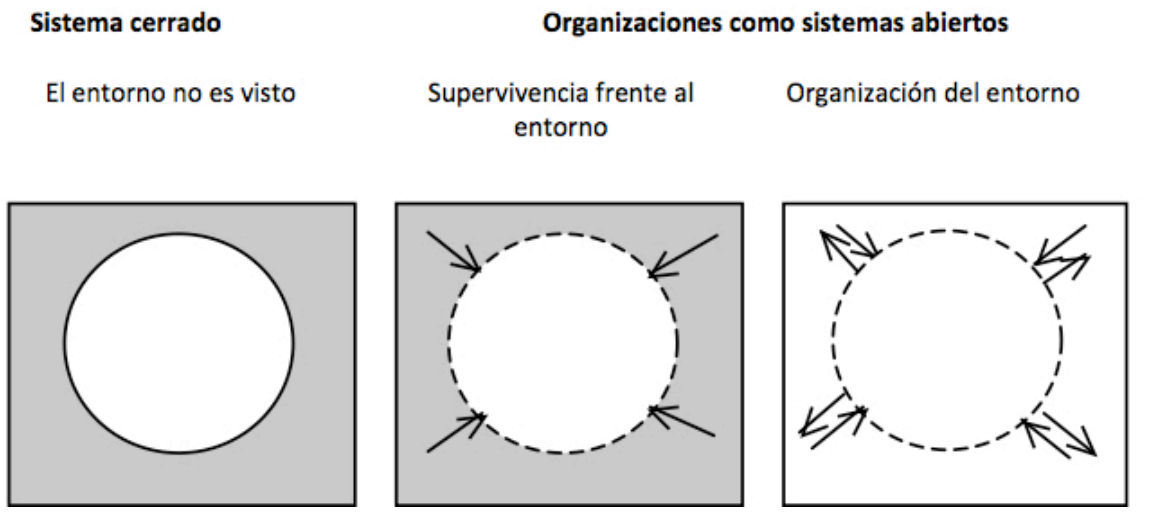

Sistemas autopoiéticos

Apertura y clausura

FUENTE: ELABORACIÓN PROPIA

Cada ilustración muestra la diferencia entre un sistema (una organización determinada o población de organizaciones) y su entorno, vale decir, todo lo demás. De izquierda a derecha: el primer gráfico representa el paradigma de sistemas cerrados. Los límites de la organización son fijos y el entorno de la organización es gris porque permanece inadvertido. El segundo gráfico representa la idea de organizaciones pasivas que en su operación luchan por sobrevivir en entornos hostiles. En este caso, el entorno no es gris porque sea pasado por alto por la organización, sino porque esta lo ve como "puro ruido". El tercer gráfico muestra el aumento de la preocupación por la "organización del medio ambiente," enfoque desde el cual el entorno es reconocido como un factor crucial en la operación de las organizaciones. Incluso más, las organizaciones no solo pueden ser conformadas, sino que ellas también gestionan activamente su entorno. El cuarto y último dibujo ilustra la noción de sistemas autopoiéticos propuesta por la teoría luhmanniana de los sistemas organizados. La diferencia principal -y fundamental- radica en el movimiento y autoproducción de los límites organizacionales. Esto muestra: (i) la clausura operativa de la organización, esto es la delimitación de la identidad y autonomía de los sistemas y (ii) acoplamiento estructural, es decir, continuas irritaciones mutuas gatilladas entre el sistema y su entorno.

Hay un aspecto adicional en el cual la teoría de Luhmann puede contribuir al momento de analizar el funcionamiento de las organizaciones: ¿Dónde están los límites de las organizaciones? Recordando a Gregory Bateson (1972), es posible decir que los límites de la organización no son parte de la organización ni de su entorno. No son definidas por el tiempo ni por el espacio demarcado entre una organización y su entorno. Más bien, un sistema es su diferencia con su entorno (Luhmann 1995).

\section{ACCIONES, COMUNICACIONES Y DECISIONES}

Los especialistas organizacionales se han referido consistentemente a la noción de acción para estudiar el modo en que las organizaciones ubican sus límites. Esto puede haber estado orientado por el análisis de la burocracia weberiano y su foco en la acción instrumental que constituyó un esfuerzo pionero en el estudio de las organizaciones por la sociología 
Repensando los límites de las organizaciones por medio de la teoría de sistemas organizacionales de

Niklas Luhmann

(Weber 1978). Para mencionar otros ejemplos, Frederick Taylor (1970) propuso que las organizaciones podrían ser reexaminadas como una suma de acciones individuales que podrían ser desmontadas para su revisión y control. Posteriormente, Talcott Parsons (1968) distinguió las organizaciones de otros tipos de sistemas sociales por actuar predominantemente orientadas al logro de metas específicas (Parsons 1950, 1968). De manera más explícita, James Thompson (1967) escribió sobre las "organizaciones en acción" para analizar las organizaciones de su tiempo, las que él describió como sistemas complejos y abiertos enfrentados a la incertidumbre, pero necesitando certidumbre (Thompson 1967: 10). Creemos que este foco en la acción de la teoría organizacional ha hecho ambiguo el rol de los límites de la organización. Estimamos que el término acción no nos permite distinguir entre lo que pertenece y lo que no pertenece a una organización, ni tampoco explicar analíticamente cuándo una organización o población de organizaciones empieza y termina.

A diferencia de las acciones que pueden ser realizadas por un individuo solo, Luhmann propuso una operación inherentemente social para dar cuenta de lo que sucede dentro y entre organizaciones: Definió la comunicación de decisiones como la operación básica que constituye a las organizaciones. Luhmann apoyó esto argumentando que una organización que solo comunica, sin ser capaz de tomar decisiones, es probable que se disuelva en interacciones flojas entre individuos. De este modo, para que las organizaciones existan y se desarrollen, es necesario que sus comunicaciones sean reconocidas y comunicadas como decisiones (Luhmann 2010).

Según Luhmann, a diferencia de los sistemas de interacción (e.g., protestas, demostraciones) o la sociedad, las organizaciones pueden comunicar fuera de sus límites por su propia cuenta. Por una parte, la sociedad comprende todas las comunicaciones posibles y, en consecuencia, no puede existir comunicación alguna afuera de sus límites. Y, aunque los sistemas de interacción pueden lograr niveles de consenso suficientes para transmitir sus demandas, las comunicaciones de estos sistemas son difíciles de recordar, trazar, o conectar a comunicaciones futuras (Luhmann 2010). Luhmann dice que, por su parte, las organizaciones pueden comunicarse con sus entornos porque están dotadas de la memoria necesaria para conectar decisiones pasadas con decisiones futuras y visualizar alternativas nuevas o no vistas previamente.

Elaborando sobre esto, Luhmann define una organización como una cadena de decisiones comunicadas, transmitidas por quienes tienen derecho a tomar decisiones. La organización asigna la responsabilidad de decidir a ciertos individuos, especificando el alcance y ámbito de su jurisdicción. Como resultado, quienes no pertenecen a la organización no pueden tomar decisiones relativas a la organización, sino solo "irritar" la dirección o forma que adoptarán dichas decisiones. 
Vamos a ampliar uno de los ejemplos empíricos de Luhmann para mostrar cómo su teoría nos ayuda a determinar los límites de las organizaciones y también para mostrar cómo se pueden proponer formas de investigar empíricamente la construcción de los límites de una organización a través del tiempo. Si uno entra a una organización, por ejemplo, a un hospital, una escuela, una galería de arte o asociación virtual, sin ser parte de ella e intenta iniciar cualquier tipo de trabajo, tal como prestar un servicio, usar una máquina, cambiar la visualización de artefactos materiales o virtuales o participar en una reunión, podría tener un éxito muy limitado. Independientemente de las características intrínsecas de la organización, pronto quedará en claro que uno no pertenece a la misma y, por lo tanto, no puede intervenir directamente en su operación (Luhmann 2010). Luhmann afirma que es la capacidad de tomar decisiones la que define qué elementos (comunicaciones) forman parte de la organización y cuáles no.

La definición de Luhmann de los límites de una organización basados en la toma de decisiones también ilumina lo que sucede en todas las organizaciones. Un ejemplo de esto son las empresas multinacionales modernas que operan principalmente a través de la contratación externa. Aunque numerosas organizaciones pueden, en última instancia, contribuir al crecimiento de una empresa importante, solo esta última es capaz de tomar decisiones respecto a su funcionamiento. Y, de la misma manera, solo sus proveedores externos pueden decidir si quieren cumplir con los requerimientos de su holding y en qué medida. Una vez más, nadie desde el exterior puede tomar decisiones respecto a lo que sucede dentro de la red de las organizaciones.

A partir de esta línea de argumentación se deduce que, a diferencia de otros enfoques de análisis, desde la perspectiva de Luhmann, ni la formalidad ni la informalidad juegan un papel especial en la construcción de los límites organizacionales. Por ejemplo, a diferencia de lo que predeciría el enfoque TCE (Transaction Cost Approach) de Oliver Williamson, la formalidad de los intercambios no tiene primacía en la determinación de la capacidad de una organización para conectarse con entidades externas. Por el contrario, las decisiones de una organización se pueden reconocer incluso sin que tengan un efecto conectivo legal. Y en contra de las teorías institucionales mencionadas anteriormente, la teoría de Luhmann sugiere que no hay ninguna razón para considerar que se dan por sentadas las prácticas culturales y los valores como el factor principal para explicar la conexión de una organización con su entorno institucional (Luhmann 2010).

Examinemos otros enfoques analíticos que se utilizan ampliamente en los estudios actuales de las organizaciones para aclarar la contribución de las propuestas de Luhmann a este respecto. El enfoque de "arraigo," (embeddedness) de Granovetter y, en general, los enfoques de redes, originalmente desarrollados en la década de los 1970s (Granovetter 1973; White 1981; Burt 1992; Uzzi 1996; Podolny \& Page 1998; Ferrary \& Granovetter 2009) proporcionan un marco adecuado para comprender la 
operación de organizaciones o arreglos de organizaciones que están en constante flujo y son de estructura compleja. Una clara ventaja de estos enfoques es que, a diferencia de los teóricos de la contingencia o institucionalistas, no solucionan la forma de la organización de acuerdo a predicciones particulares. Además de esto, mientras que los enfoques de redes podrían ayudarnos a superar estas barreras analíticas, no parecen responder a la pregunta que nos planteamos al comienzo de esta sección: ¿qué pertenece a una organización y qué no?; ¿dónde están los límites de las organizaciones? Desde este enfoque analítico, cualquier cosa puede ser parte de una red organizacional. A diferencia de la teoría de Luhmann, por lo tanto, la metáfora de la "organización sin límites" puede ser apoyada desde una perspectiva de redes.

Sostenemos que el marco conceptual de "arraigo" ofrece una mirada perspicaz para la comprensión de formas complejas de organización $\mathrm{y}$, lo que es más importante, para el estudio empírico de la formación y desarrollo de redes de organizaciones. No obstante, estimamos que, desde un punto de vista teórico, este enfoque simplifica en exceso el rol de los límites de la organización en la mantención y reproducción de los sistemas organizacionales. Si, en su lugar, consideramos la teoría de Luhmann como nuestro punto de partida analítico, nos daremos cuenta de que la formación de redes se extiende mediante acoplamientos estructurales que respetan los límites organizacionales, debido a que cada sistema es un agente particular con la capacidad de generar su propia reproducción (vale decir, la clausura operativa).

Desde el enfoque de Luhmann, por lo tanto, el hecho de que las organizaciones busquen una relación simbiótica1 con otras organizaciones, de ninguna manera reduce la importancia de los límites de las organizaciones. Como hemos visto, según Luhmann, las organizaciones son a la vez abiertas y cerradas. Debido a esto, las organizaciones son capaces de comunicarse con su entorno, precisamente porque son unidades distinguibles unas de otras, sin importar lo complejas o maleables que sean.

\footnotetext{
${ }^{1}$ Si recordamos los casos de simbiosis de la biología, veremos que el régimen de simbiosis consiste en una forma de acoplamiento estructural entre sistemas que mantienen su identidad y no pasan a ser parte uno del otro.
} 
FIGURA 2: ENFOQUES DE REDES VERSUS SISTEMAS AUTOPOIÉTICOS DE LUHMANN

\section{Enfoque de arraigo \\ (embeddedness)}

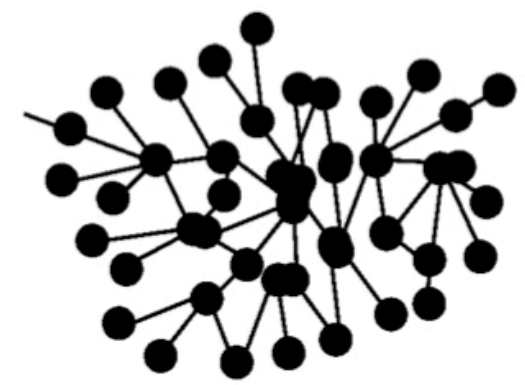

Enfoque de Luhmann

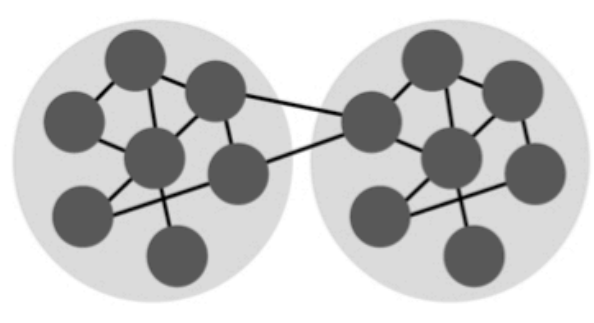

FUENTE: ELABORACIÓN PROPIA

La figura de la izquierda tiene por objeto representar el "enfoque de arraigo" o enfoques de redes en general, los cuales consisten, fundamentalmente, de nodos (es decir, individuos, organizaciones o poblaciones de organizaciones) conectadas por aristas. La figura de la derecha ilustra el enfoque de Luhmann, según el cual los límites de las organizaciones no pueden darse por sentado. A diferencia del enfoque de Luhmann, la metáfora de la "organización sin límites" puede ser sostenida desde un enfoque analítico de redes.

Tal como se representa en la figura 2, creemos que la teoría de Luhmann ofrece una plataforma teórica mejorada en comparación con el enfoque de "arraigo" en particular o en general de enfoques de redes, con respecto a reconocer analíticamente los límites de las organizaciones, es decir, el punto en el que las organizaciones comienzan y terminan. Esto es especialmente relevante en la sociedad contemporánea, dado que los límites de las organizaciones se han vuelto más flexibles $\mathrm{y}$, por lo tanto, más difíciles de distinguir. En nuestros días, los cambios se aceleran, del mismo modo que la necesidad de las organizaciones de redefinir sus límites. Sin embargo, el hecho de que las formas complejas y acopladas flojamente de organización estén ganando predominio no significa que los límites de los sistemas se estén borrando gradualmente o que los límites de las organizaciones estén perdiendo su importancia. Ahora más que nunca, las organizaciones necesitan mantener sus límites para ser capaces de gestionar y anticipar los cambios en sus entornos.

Stefan Kühl (2016) advierte contra los peligros de las jerarquías aplanadas y la desatención de los límites. Las organizaciones reaccionaron a la rápida transformación de los mercados y a los adelantos tecnológicos con grupos de trabajo (workshops, task forces) que juntaban a empleados de diferentes roles funcionales. Los proyectos de grupo comenzaron a completar o reemplazar crecientemente a las jerarquías funcionales. Se puede observar las experiencias pasadas de compañías, cuerpos administrativos y organizaciones políticas con el derrumbe de sus jerarquías y los 
Repensando los límites de las organizaciones por medio de la teoría de sistemas organizacionales de

Niklas Luhmann

esfuerzos por la descentralización. Parecería que el impulso a romper todos los límites, tanto internos como externos, amenaza con hacer difusa a toda la organización, privando a las organizaciones de su cohesión interna. Esto conduce a tres dilemas de nuevo cuño:

1. Los empleados pierden su imagen clara de la organización (dilema de la identidad).

2. Las organizaciones dedicadas a la innovación y el cambio crean zonas de inseguridad dentro de su organización, las que amenazan con impulsar una "politización permanente" de los procesos internos y la toma de decisiones (dilema de la politización).

3. En reacción a la complejización, cada vez más organizaciones recurren a estrategias simplificadoras, pero es precisamente este impulso a la simplificación de procesos el que conduce a una complejidad creciente, aunque no sea percibida como tal (dilema de la complejidad).

\section{CONSENSO, CONFLICTO Y COORDINACIÓN}

Hay un último aspecto del enfoque de Luhmann que debemos considerar en nuestro esfuerzo por mostrar cómo su teoría puede contribuir a la investigación contemporánea de organizaciones: el rol de los conflictos y contradicciones. Luhmann sugiere que, dado que la operación básica de las organizaciones es la comunicación de decisiones, cada selección de una comunicación incluye su contradicción. Y cada vez que una comunicación es negada y este rechazo se comunica, surge el conflicto. De esta manera, el conflicto y las contradicciones están siempre latentes en la operación de las organizaciones. Sobre esta base, Luhmann afirma que lo importante para el mantenimiento de los límites de las organizaciones no son la armonía ni la disonancia por sí mismas, sino la coordinación. Según Luhmann, el conflicto es tan capaz como el consenso de conectar las decisiones anteriores con las decisiones posteriores $\mathrm{y}$, de ese modo, de garantizar el mantenimiento de los límites de una organización.

$\mathrm{Al}$ enfocar su mirada en la coordinación, en lugar del acuerdo o consenso, Luhmann aclara aspectos importantes del funcionamiento de las organizaciones. A diferencia de los modelos de sistemas de Thompson o de Parsons anteriormente mencionados, en el enfoque de Luhmann, los entornos enfrentados por las organizaciones no son vistos como inevitablemente turbulentos o impredecibles, ni son los entornos vistos como algo que necesariamente evoluciona hacia una mayor estabilidad y orden, como parecen predecir las teorías institucionales. Los modelos de sistemas abiertos no pueden reconocer cómo las organizaciones logran movilizar y explotar valores en conflicto, con el fin de ser capaces de colocar sus límites y perdurar. Luhmann explica que los valores son esencialmente proposiciones de sentido que las organizaciones seleccionan con el 
fin de tomar decisiones. En este proceso, los valores pueden (y deben) ser aceptados o rechazados por las organizaciones. Por lo tanto, la adopción de una orientación de valor particular por una organización, en un momento dado, incluye necesariamente un conflicto entre los diferentes órdenes de valor (Luhmann 2010). Con respecto a esto, la teoría de Luhmann nos recuerda que, aunque meta-valores más o menos estables coincidan al interior o entre sistemas organizacionales, muchos valores diferentes que compiten entre sí necesariamente operan en la definición que hacen las organizaciones de sus límites. Como resultado, en contra de los enfoques institucionales, Luhmann pone de manifiesto que la base de la legitimidad puede variar mucho de una organización a otra, así como de una red de organizaciones a otra, dependiendo de cada situación (Luhmann 2010). En resumen, el orden se genera en y por las organizaciones, pero este es orden al borde del caos (Fontdevila, Opazo \& White 2011).

En esta perspectiva, la teoría de Luhmann se corresponde con una serie de estudios empíricos de las organizaciones contemporáneas. A través de la investigación etnográfica en empresas de Silicon Valley en Nueva York, David Stark (Girard \& Stark 2003; Stark 2009) muestra cómo múltiples principios de legitimación (orders of worth) coexisten simultáneamente en organizaciones y se reordenan constantemente dentro de las organizaciones, con el fin de que estas puedan sobrevivir y perdurar. De manera semejante a la teoría de Luhmann, Stark (2009) llega a la conclusión de que uno de los principales desafíos de las organizaciones contemporáneas es pasar de la coordinación por conocimientos compartidos a la coordinación por malentendidos. A un nivel más amplio, un estudio de Brian Uzzi (1996) sobre tiendas de ropa en Nueva York encontró que diversas lógicas operan dentro del sistema de intercambio interorganizacional de las empresas. Las empresas pueden decidir unirse a una red basada en principios diferentes - o incluso contradictorios - tales como aumentar sus posibilidades de supervivencia, adquirir información detallada, conseguir un estatus más alto o asegurar mayores beneficios.

En suma, estos estudios, en conjunto con el enfoque de Luhmann, ofrecen un marco conceptual analítico que permite que pretensiones normativas competidoras sean parte de la formación y desarrollo de una organización o redes de organizaciones. Estimamos que este tipo de enfoques presenta una solución a la inconmensurabilidad de las lógicas institucionales que se han encontrado estar en juego dentro y a través de las organizaciones contemporáneas. Esta línea de argumentación puede ayudarnos a enfrentar la cuestión de cómo se logra la coordinación en, por y entre las organizaciones ante la multiplicidad de sistemas de valores que funcionan en la sociedad contemporánea. Dado que las organizaciones son operacionalmente cerradas, y definen sus propios mapas de sus entornos, su participación en las redes necesariamente ocurre como una "coordinación pragmática de opacidades" (Rodríguez \& Torres 2007). 
Repensando los límites de las organizaciones por medio de la teoría de sistemas organizacionales de

Niklas Luhmann

\section{CONCLUSIÓN}

Hemos esbozado una serie de aportes que la teoría de Luhmann puede ofrecer a la teoría e investigación contemporáneas de los límites organizacionales. En primer lugar, hemos señalado cómo la distinción básica de Luhmann entre organización/entorno afirma que los procesos de construcción de límites son el mecanismo clave que explica la existencia y la resistencia de una organización. En segundo lugar, hemos descrito cómo el concepto de autopoiesis de Luhmann puede abrir avenidas para reexaminar las implicaciones y limitaciones del "paradigma de sistemas abiertos" que todavía domina en la teoría organizacional contemporánea (Scott \& Davis 2003). Puede hacerlo, hemos indicado, resaltando el funcionamiento cerrado y abierto de los sistemas organizacionales. En tercer lugar, señalamos que el énfasis de Luhmann sobre la comunicación de decisiones como el principal elemento que constituye a las organizaciones puede permitirnos distinguir mejor los límites de las organizaciones o poblaciones de organizaciones. Por último, hemos explicado cómo en línea con estudios organizacionales contemporáneos, el foco de Luhmann en la coordinación, como opuesta al orden o el desorden en sí mismos, puede ampliar nuestro conocimiento acerca de cómo las organizaciones se desarrollan, explotando lógicas institucionales diferentes dentro de un solo dominio o entre dominios. Si bien todos estos elementos de la teoría de Luhmann tienen el potencial de arrojar luz sobre los análisis contemporáneos de organizaciones, creemos que es necesaria una amplia investigación empírica para evaluar el grado en que el enfoque de Luhmann puede ayudar a abordar estas cuestiones.

Concluimos proponiendo dos formas en que la obra de Luhmann puede iluminar futuras investigaciones acerca de las organizaciones. Al afirmar que la comunicación es el componente central de las organizaciones, sugerimos que el marco conceptual de Luhmann es particularmente adecuado para la investigación que tiene por objeto examinar la función de una organización sobre la base de sus comunicaciones, en lugar de mediante la observación de las actividades de la vida real, los objetivos, y las rutinas de los distintos miembros de una organización. Este es el caso de muchos estudios empíricos de organizaciones realizados en retrospectiva; casos en los que los investigadores no pueden seguir a los miembros de la organización en acción, sino que, mediante el uso de datos secundarios, teniendo acceso a la "vida" de la organización solo a través de las comunicaciones transmitidas en y por la organización y que han quedado registradas. Son ejemplos de este tipo de estudios las investigaciones de la organización de la NASA, conducidas por Diane Vaughan $(1996,1998)$ y el estudio de Anteby y Molnar (2012) sobre la organización aeronáutica "Sneema." En ambos casos, los autores utilizan las comunicaciones organizacionales como la principal fuente de datos para comprender aspectos específicos de la vida de una organización. Mientras que Vaughan se basa 
en los archivos históricos como la ventana principal para "ver" cómo se tomó la decisión de lanzamiento del Challenger en la NASA, Anteby y Molnar se centran en los boletines internos de Snecma con el fin de examinar la supervivencia de la empresa durante un período de 50 años. Proponemos que el foco de Luhmann sobre las comunicaciones y decisiones para entender cómo perduran las organizaciones podría ser muy útil para este tipo de análisis histórico de las organizaciones.

Por otra parte, la importancia de la dinámica entre una organización y su entorno presupuesta en la teoría de Luhmann puede ofrecer posibilidades alternativas interesantes para el estudio de las relaciones de poder, un tema que, en repetidas ocasiones, ha demostrado ser fundamental para el estudio de las organizaciones (por ejemplo, Perrow 1986, Coleman 1974). Luhmann describe a las organizaciones como sistemas complejos dotados de la capacidad para establecer sus límites respecto a su entorno. A partir de esto, una manera en que el marco conceptual de Luhmann puede orientar el estudio de la dinámica del poder consiste en la forma de simples "decisiones comunicadas" (es decir, la producción de diferencias) que operan a nivel discursivo en el curso del desarrollo de las organizaciones. La dinámica de la toma de decisiones organizacionales descrita por Luhmann es informativa y detallada. Estudios empíricos podrían explorar esta dinámica dentro y a través de organizaciones tratando, por ejemplo, una organización como el sistema focal y analizar el contenido de los intercambios comunicativos en lo que se refiere a variables independientes pertinentes, tanto dentro como fuera de la organización. Este enfoque podría impulsar a los investigadores a dejar de lado ideas preconcebidas acerca de las fuerzas sociales que podrían dirigir las relaciones dentro y entre organizaciones $y$, en su lugar, alentar investigaciones que permitan emerger, de los mismos análisis, factores guías novedosos e inesperados.RM

\section{AGRADECIMIENTOS}

Agradecemos especialmente a Corinne Kirchner, David Stark, Diane Vaughan y Harrison White por sus profundos comentarios a versiones anteriores. Muchas gracias a Fabien Accominotti, Victor Corona, Natan Dotan, Rajiv Nunna y Jason Widabi por sus constructivas sugerencias. Versiones tempranas de este artículo fueron presentadas en SASE 23rd Annual Conference, Universidad Autonoma, Madrid, 2011, en la III WCSA Conference Vienna, 2012 y en diversos talleres de la Universidad de Columbia.

\section{REFERENCIAS}

Anteby, M. \& Molnar, V. (2012). Collective Memory Meets Organizational Identity: Remembering to Forget in a Firm's Rethorical History. Academy of Management Journal, 55(3), 515-540.

Ashkenas, R., Ulrich, D., Jick, T. \& Kerr, S. (2002). The Boundaryless Organization: Breaking the Chains of Organization Structure. San Francisco: Jossey-Bass. Bateson, G. (1972). Steps to an Ecology of Mind. New York: Chandler Publishing. Burns, T. \& Stalker G. (1961). The Management of Innovation. London: Tavistock. 
Repensando los límites de las organizaciones por medio de la teoría de sistemas organizacionales de Niklas Luhmann

Burt, R. (1992). The Social Structure of Competition. In: N, Nitin \& R. Eccles, Networks and Organizations (pp. 57-91). Boston: Harvard Business School Press.

Brown, S. \& Eisenhardt K. (1998). Competing on the Edge: Strategy as Structured Chaos. Boston: Harvard Business School Press.

Carroll, G. \& Hannan, M.T. (2000). Density-Dependent Processes. Reprinted in: M. Handel (Ed.), The Sociology of Organizations, 2003 (pp. 254-261). Thousand Oaks: Sage.

Coleman, J. (1974). Power and the Structure of Society. New York: Norton.

DiMaggio, P. \& Powell, W. (1983). The Iron Cage Revisited: Institutional Isomorphism and Collective Rationality in Organizational Fields. American Sociological Review, 48(2), 147-160.

Elyachar, J. (2005). Markets of Dispossession: NGOs, Economic Development, and the State in Cairo. Durham: Duke University Press.

Febbrajo, A. \& Harste G. (Eds.). (2013). Law and Intersystemic Communication. Understanding 'Structural Coupling'. Surrey: Ashgate.

Ferrary, M. \& Granovetter, M. (2009). The Role of Venture Capital Firms in Silicon Valley's Complex Innovation Network. Economy and Society, 38(2), 326-359.

Fontdevila, J. Opazo, M. \& White H. (2011). Order at the Edge of Chaos: Meanings from Netdom Switchings Across Functional Systems. Sociological Theory, 29(3), 178-198.

Gieryn, T. (1983). Boundary-Work and the Demarcation of Science from Non-science: Strains and Interests in Professional Interests of Scientists. American Sociological Review, 48, 781-95.

Girard, M. \& Stark D. (2003). Heterarchies of Value in Manhattan-based New Media Firms. Theory, Culture \& Society, 20(3), 77-105.

Granovetter, M. (1985). Economic Action and Social Structure: The Problem of Embeddedness. American Journal of Sociology, 91(3), 481-510.

Granovetter, M. (1973). The Strength of Weak Ties. American Journal of Sociology, 78(6), 1360-1380.

Hannan, M. \& Freeman J. (1977). The Population Ecology of Organizations. American Journal of Sociology, 82(5), 929-964.

Handel, M. (Ed.) (2003). The Sociology of Organizations: Classic, Contemporary and Critical Readings. Thousand Oaks: Sage.

Hannan, M. \& Freeman, J. (1993). Organizational Ecology. Cambridge: Harvard University Press.

Hernes T. \& Bakken, T. (2003). Implications of Self-reference: Niklas Luhmann's Autopoiesis and Organization Studies. Organization Studies, 24(9), 1511-1536.

Kühl, S. (2016). When the Monkeys Run the Zoo. Texto inédito, última revisión.

Christel, L. \& Bachmann, R. (2002). Trust Within and Between Organizations: Conceptual Issues and Empirical Applications. New York: Oxford University Press.

Luhmann, N. (1981). The Improbability of Communication. International Social Sciences Journal, 32(1), 122-132.

Luhmann, N. (1994). What is the Case? And What Lies Behind It? The Two Sociologies and the Theory of Society. Sociological Theory, 12(2), 126-139.

Luhmann, N. (1995). Social Systems. Palo Alto: Stanford University Press. [1984]

Luhmann, N. (2010). Organización y decisión. Herder: México. [2000]

Luhmann, N. (2007). La sociedad de la sociedad. Ciudad de México, México: Herder.

MacKenzie, D., Muniesa, F. \& Siu, L. (Eds.). (2007). Do Economists Make Markets? On the Performativity of Economics. Princeton: Princeton University Press. 
Maturana, H. \& Varela, F. (1998). The Tree of Knowledge: The Biological Roots of Human Understanding. Boston: Shambhala. [1984]

Meyer, J. \& Rowan B. (1977). Institutionalized Organizations: Formal Structure as Myth and Ceremony. American Journal of Sociology, 83(2), 340-363.

Mohe, M \& Seidl, D. (2011). Theorizing the client-consultant relationship from the perspective of social-systems theory. Organization, 18(1), 3-22.

Padgett, J. \& Powell, W. (2012). The Emergence of Organizations and Markets. Princeton: Princeton University Press.

Parsons, T. (1956). Suggestions for a Sociological Approach to the Theory of Organizations-I. Administrative Science Quaterly, 1(1), 63-85.

Parsons, T. (1968). La estructura de la acción social. Madrid: Ediciones Guadarrama.

Perrow, C. (1986). Complex Organizations: A Critical Essay. New York: Random House. [1972]

Podolny, J. \& Page, K. (1998). Network Forms of Organization. Annual Review of Sociology, 24, 57-76.

Powell, W. (1990). Neither Market nor Hierarchy: Network Forms of Organization. Research on Organizational Behavior, 12, 295-336.

Powell, W. \& DiMaggio, P. (Eds.) (1991). The New Institutionalism in Organizational Analysis. Chicago: The University of Chicago Press.

Pfeffer, J. \& Aldrich, H. (1976). Environments of Organizations. Annual Review of Sociology, 2, 79-105

Pfeffer, J. \& Salancik, G. (1978). The External Control of Organizations: A Resource Dependence Perspective. In M. Handel (Ed.), The Sociology of Organizations, 2003 (pp. 233-242). Thousand Oaks: Sage.

Rodríguez, D. \& Torres, J. (2007). El derecho de la sociología de la sociedad. In: Artur Stamford da Silva (Ed.), Sociologia do direito (pp. 135-201). Curitiba: Juruá.

Schreyögg, G. \& J. Sydow. (2010). Understanding Organization as Process: Theory for a Tangled World. London: Routledge.

Scott, W. \& Davis, G. (2003). Organizations and Organizing: Rational, Natural and Open System Perspectives. Upper Saddle River: Pearson Education.

Scott, W. (2004). Reflections of Half a Century of Organizational Sociology. Annual Review of Sociology, 30, 1-21.

Seidl, D. \& Becker, K. (Eds.). (2005). Niklas Luhmann and Organization Studies. Malmo: Liber \& Copenhagen Business School Press.

Seidl, D. (2005). Organisational Identity and Self-transformation. An Autopoietic Perspective. Aldershot: Ashgate.

Seidl, D. \& Becker, K. (2006). Organisations as Distinction Generating and Processing Systems. Niklas Luhmann's Contribution to Organisation Studies. Organization, 13(1), 9-35.

Selznick, P. (1953). TVA and the Grass Roots: A Study in the Sociology of Formal Organization. Los Angeles: California Press. [1949]

Senge, P. (1990). Fifth Discipline: The Art and Practice of the Learning Organization. New York: Doubleday.

Spencer-Brown, G. (1979). Laws of Form. New York: Dutton.

Stark, D. (2009). The Sense of Dissonance: Accounts of Worth in Economic Life. Princeton: Princeton University Press.

Taylor, F. (1970). Principles of Management. New York: Harper. [1911]

Thompson, J. (1967). Organizations in Action. New York: Mc Graw Hill.

Vaughan, D. (1996). The Challenger Launch Decision: Risk Technology, Culture and Deviance at NASA. Chicago: University of Chicago Press.

Vaughan, D. (1998). Rational Choice, Situated Action, and Social Control: The Challenger Launch Decision. Law and Society, 1, 23-61.

Von Foerster, H. (1981). Observing Systems. California: Seaside. 
Repensando los límites de las organizaciones por medio de la teoría de sistemas organizacionales de Niklas Luhmann

Uzzi, B. (1996). The Sources and Consequences of Embeddeness for the Economic Performance of Organizations: The Network Effect. American Sociological Review, 61, 674-689

Weber, M. (1947). The Theory of Social and Economic Organization. New York: Oxford University Press.

Weber, M. (1878). Economy and Society: An Outline of Interpretive Sociology. Los Angeles: University of California Press.

Weick, K. (1979). The Social Psychology of Organizing. New York: Random House. [1969]

Weick, K. (1995). Sensemaking in Organizations. London: Sage.

White, H. (1981). Where do Markets Come from? American Journal of Sociology, $87(3), 517-547$.

White, H. (1992). Identity and Control: A Structural Theory of Social Action. Princeton: Princeton University Press.

White, H. (2008). Identity and Control: How Social Formations Emerge. Princeton: Princeton University Press.

Williamson, O. (1981). The Economics of Organizations: The Transaction Cost Approach. American Journal of Sociology, 87(3), 548-577.

Williamson, O. \& William G. (1981). The Markets and Hierarchies and Visible Hand Perspectives. In: A. Van de Veny \& W. Joyce (Eds.), Perspectives on Organization Design and Behavior (pp. 347-370). New York: Wiley.

\section{SOBRE LOS AUTORES}

María Pilar Opazo es Profesora Auxiliar Adjunta e Investigadora postdoctoral en la Escuela de Negocios de la Universidad de Columbia, Nueva York. PhD y M.A. en Sociología por la misma casa de Estudios y Socióloga por la Pontificia Universidad Católica de Chile. Entre sus áreas de especialización se encuentran: Sociologia Organizacional y Estudios de Innovación. Entre sus últimas publicaciones destacan Appetite for Innovation Creativity and Change at elBulli (2016) y Order at the Edge of Chaos: Meanings from Netdom Switchings Across Functional Systems (en coautoría con Harrison C. White y Jorge Fontdevila, 2011)

Darío Rodríguez Mansilla es profesor titular de la Escuela de Ingeniería Industrial, perteneciente a la Universidad Diego Portales, Chile. Doctor en Sociología por la Universidad de Bielefeld, Alemania. Entre sus áreas de especialización se encuentran: Teoría de Sistemas y Teoría de la Organización. Entre sus publicaciones más destacadas se cuentan: (en coautoría con Javier Torres Nafarrate) Introducción a la Teoría de la Sociedad de Niklas Luhmann (Herder-Universidad Iberoamericana, 2008), Gestión Organizacional. Elementos para su estudio (Ediciones Universidad Católica de Chile, 2001).

CONTACTO

Columbia University

Columbia Bussines School

3022 Broadway, New York,

NY 10027 212-854-5553

mpo2110@columbia.edu

Recibido: octubre 2016

Aceptado: febrero 2017 